\title{
Immune Therapy in Autism: Historical Experience and Future Directions with Immunomodulatory Therapy
}

\author{
Michael G. Chez* and Natalie Guido-Estrada ${ }^{\dagger}$ \\ *Pediatric Neurology and Autism Neurodevelopmental Program, Sutter Neuroscience Institute, Sacramento, California 95816, \\ and ${ }^{\dagger}$ Department of Neurology, University of California Davis School of Medicine, Sacramento, California 95817
}

Summary: Autism affects 1 in 110 new births, and it has no single etiology with uniform agreement. This has a significant impact on the quality of life for individuals who have been diagnosed with autism. Although autism has a spectrum quality with a shared diagnosis, it presents a uniquely different clinical appearance in each individual. Recent research of suspected immunological factors have provided more support for a probable immunological process or for processes that may play a role in the acquisition of an autistic condition. These factors include prenatal, genetic, and postnatal findings, as well as the discovery of a dysfunctional chronic pro-inflammatory state in brain tissue and cerebrospinal fluid in subsets of autistic patients. These findings offer new theories that may lead to the development of disease modification or preventative therapeutic options in the near future. This article reviews prenatal, genetic, and observed immune aspects of the autism condition that may be risk factors in the presentation of the autistic clinical phenotype. Historical immune interventions in autism are reviewed and potential new therapies and interventions are discussed. Key Words: Autism, immune therapy, immune dysfunction, autoimmunity.

\section{PART 1: IMMUNE SYSTEM AND AUTISM: DEFINING THE RELATIONSHIP}

Autism is currently the leading cause of developmental disability in the United States and most other countries of the world. This condition currently affects 1 in 110 children born in the United States as of $2009 .^{1}$ Autism is best defined as a spectrum of heterogeneous developmental disabilities, which mainly involves three core aspects of behaviors: 1) speech and communication, 2) social interest and interaction, and 3) stereotypic behaviors or mannerisms. ${ }^{2}$ Historically, the incidence of autism has increased; however, debate exists as to whether this reflects the simple population growth, re-categorization, and increased recognition, or whether there is a true increase in percent of the population affected. ${ }^{3}$ Despite this controversy, most experts and the general population agree that the incidence of autism has greatly increased, especially in states such as California, which has shown a massive increase of autistic children in state educational records for the last 2 decades. $^{4-6}$

Address correspondence and reprint requests to: Michael G. Chez, M.D., 2800 L Street, Suite 340, Sacramento, CA 95816. E-mail: chezm2@sutterhealth.org.
Current thinking is that multiple risk factors may need to occur for autism to exist, and these factors may have various underlying combinations in different subgroups. Some factors may be familial cases, and others are clearly environmental, such as extreme prematurity or infection. Epilepsy occurs in children with autism. Conversely, autistic behaviors can occur in children with severe early onset epilepsy. ${ }^{7,8}$ Genetic conditions may also be associated with autism, but autism may be one feature of the condition that has other problems as well (e.g., tuberous sclerosis or fragile X syndrome) ${ }^{9}{ }^{910}$ Some genes, such as the tyrosine kinase receptor $7 \mathrm{q} 31$ metastasis receptor site (MET) gene, an immune related gene affecting tyrosine kinase that can be involved in CNS immune dysfunction, can double the risk of autism. ${ }^{11}$ Prenatal factors, such as maternal immune history and in utero environments, may historically offer insight into the immune role in autism as well. ${ }^{12}$ Recent research on postnatal immune issues identify various serum antibodies, as well as cord blood neurotrophins and cytokine levels as potential factors. ${ }^{12-17}$ Atladottir et al. ${ }^{18}$ found an increased risk of ASDs in children with a maternal history of rheumatoid arthritis and celiac disease. Also, increased risk of infantile autism was observed for chil- 
dren with a family history of type 1 diabetes. These risk factors and others will be further discussed as follows.

\section{Historical observations}

Patients who have had neonatal herpes simplex, measles rubella, or congenital infections, such as toxoplasmosis or cytomegalovirus (CMV), in many cases have suffered brain injury and often have autistic traits. In fact, 1 in 1000 patients with measles infection involving the CNS has a subsequent diagnosis of autism. These observations have made it clear that in some cases autism may be acquired after infection. ${ }^{12}$

From the late 1980s onward, hypotheses on the relationship of the immune system to the etiology of autism began to emerge. ${ }^{19-21}$ Several scientific studies began to consider possible auto-antibodies that were noted in autistic populations. ${ }^{13,16,22-24}$ Observations were also made on the relationship of familial autoimmune histories in patients with autism. Many authors have described autism clusters in families with high incidence of autoimmune disorders, such as Hashimotos thyroiditis, Crohn's disease, diabetes type 1, rheumatoid arthritis, other immune disorders, and certain human leukocyte antigen (HLA) haplotypes. ${ }^{19-21}$

\section{Auto-antibodies in the autism spectrum}

There is emerging evidence for the production of antibodies against brain tissue in autistic patients. Nonspecific whole brain antibodies in the serum of patients with autism were described by Todd et al. ${ }^{16}$ Another author, Singh et al., ${ }^{23}$ described anti-myelin antibodies in the serum of patients with autism. ${ }^{24} \mathrm{He}$ also described higher antibody titers against measles virus from measlesmumps-rubella (MMR) vaccinations suggesting an overly robust immune response may be present. Connolly ${ }^{22}$ and Connolly et al. ${ }^{13}$ described anti-endothelial brain capillary antibodies in the serum of autistic patients with regression, which also cross reacted with anti-brain derived neurotrophic factor antibodies, as well. These patients also often had an abnormal clinical EEG even in the absence of clinical epilepsy. ${ }^{13,22}$ In addition, placental and umbilical cord blood samples show abnormal brain-derived neurotrophic factor levels, as well as other neuropeptide levels in autism and cerebral palsy, but not mental retardation cases. ${ }^{14}$

Evidence of dysfunction in the immune system of the autistic patient has been cited in multiple articles. Dysfunction in suppressor T-cells was noted by Warren et al., ${ }^{25}$ whereas Stubbs and Crawford ${ }^{26}$ described suppressed lymphocyte function. Other immune abnormalities were observed by Margaretten and Warren, ${ }^{27}$ including decreased complement $\mathrm{C} 4$ levels in serum, and decreased natural killer cell function in serum samples from autistic individuals.

\section{Immunoglobulin levels and autism}

Decreased immunoglobulin levels have also been studied in conjunction with autism. Dysfunctional low total immunoglobulin IgG levels and low levels of IgG subclasses have been described by Gupta et al. ${ }^{28}$ and others. ${ }^{29,30}$ Decreased levels of IgA and low IgA conditions, such as ataxia telangiectasia have been found to be associated with autism. Patients with hypogammaglobulinemia in conditions like DiGeorge syndrome have also been noted to display autistic traits. Ten percent of autistic patients with low IgG or IgG subclasses who underwent intravenous gamma globulin infusions recorded improvements in regressive autistic traits. ${ }^{31,32}$ Treatments for underlying immune conditions will be further detailed and discussed later in the article.

\section{Genetics of autism and immunological risk factors}

Findings suggest the existence of 15 to 22 different links to autism risk, with no single etiology found, but genetics clearly play a role. ${ }^{9,33}$ Monozygotic twins share an autistic spectrum phenotype $50 \%$ to $90 \%$ of the time, whereas dizygotic twins are often far less frequently involved at only $0 \%$ to $24 \%$ of the time..$^{34-36}$ Siblings of autistic patients have 5 to 10 times the risk of having autism compared to the general population. ${ }^{33}$ Despite these genetic ties to autism risk, other factors such as familial autoimmunity, maternal intra-uterine environment, and perhaps other stressors, such as infection or postnatal risk factors may play a more significant role in defining the risk for developing autism. Genetic mutations in phosphatase and tensin homolog (PTEN) gene are associated with cases of lymphoid hyperplasia and cases of macrocephaly. ${ }^{37}$ These types of observations strengthen the multifactoral theory on risk factors for developing autism, especially regarding immunological variables. $^{38}$ Some MET allelic variants or MET gene mutations may double the risk of autism and effect tyrosine kinase, which may play a role in innate immune cytokine function among other variables. ${ }^{11}$ Many of the genetic findings have some degree of critical relationship to immune function, the role of immune factors to neuronal migration, or neurochemical receptors and cellular immune regulation. ${ }^{39,40}$ Prior work by others have noted that maternal HLA haplotype genetics are more prone to autoimmune variants. ${ }^{21}$

\section{Immune models in animals}

Animal studies have the potential to identify conditions that may cause autism, especially intrauterine exposure models and early developmental exposure to various immune dysregulatory factors. In one model, pregnant mice that were exposed to influenza virus bore offspring that exhibited some symptoms of autistic-like behavior when examined at an adult age. ${ }^{12}$ Subsequently, animal models have been looked at for mimicking bacterial or viral infections, and these models have shown abnormal 
changes similar to actual infections; for example, these included models that have shown abnormal pathology in Purkinje cells. ${ }^{41,42}$ Several animal models of fetal manipulation in rats and mice via induced inflammatory response in utero have mimicked autistic and schizophrenia symptoms. ${ }^{41,43}$ Recent work has focused on the roles of cytokines and pro-inflammatory effects of tumor necrosis factor (TNF)- $\alpha$, interleukin- 6 , and interleukin$1 \beta{ }^{12,41-45}$ Interleukin-10 appears to modulate more protection in animal models. ${ }^{46}$ These models may reflect the same process of chronic activation of pro-inflammatory cytokines in brain tissue and cerebrospinal fluids. This may reflect human autism models of brain tissue and cerebrospinal fluids showing chronic activation of proinflammatory cytokines. ${ }^{15,17,47}$

Human sera containing immune auto-antibodies have been found in mothers with autistic offspring. Transfer of these serum antibodies to pregnant mice or rats has caused offspring to elicit behaviors similar to autistic humans. ${ }^{48-51}$ Some studies have shown auto-antibodies in children with autistic regression, which suggest persistent inflammatory processes as well. ${ }^{13,22}$ Both in utero maternal immune transfer of auto-antibodies against the fetal brain and induction of cytokine activation toward a pro-inflammatory response have been demonstrated in pregnant mice. ${ }^{49}$ These studies strongly suggest an increased risk of the in utero process, but the effect of which may not present until later in development when certain neuronal interconnections or excitatory learning potentiation occur during synaptogenesis. This auto-antibody connection may be most active when children undergo language and social development between the ages of 12 and 24 months, which correlates with the ages when most regression is observed to occur. ${ }^{12,41,42,45}$

Pregnancy normally results in some degree of elevated maternal immune systemic inflammation. However, exposure to pro-inflammatory inducers may trigger abnormally exaggerated inflammation. This may be why mothers with autoimmune conditions like psoriasis or asthma show increased rates of autistic offspring. ${ }^{52}$ Obviously, autoimmune disease in mothers or close relatives may be clinically relevant in the future to determine which children with autism may respond best to future immunetargeted therapies.

\section{Human evidence of ongoing atypical inflammatory response}

There is evidence that patients with autism may have serum markers for inflammation, including elevation of some cytokines, auto-antibodies, and lower levels of normal immunoglobulins for immune defense. ${ }^{13,15,17,22,53}$ Maternal transfer of immune dysregulation also is inferred by the fact that 47 of 100 mothers with serum auto-antibodies (especially at the $37 \mathrm{kD}$ site) had offspring diagnosed with regressive cases of autism. ${ }^{30,48}$
This evidence suggests that mothers positive for autoantibodies have almost a 50\% chance of having children with the specific regressive phenotype of autism, indicating a potential delay from in utero exposure to clinical onset. This subgroup of autistic children may be candidates for treatment or modulation of their autism through future immune manipulation, if standardized screening tests can be successfully developed.

More recently, brain tissue and CSF cytokine elevations have been observed in human patients. Elevation, particularly of interleukin-6 and also of other pro-inflammatory markers in the frontal cingulated cortex and CSF, has been described by Vargas et al. ${ }^{17}$ Patients with regressive autism studied between pre-school age and 12 years old have been shown to have elevated CSF:serum ratios of TNF- $\alpha$, interleukin (IL)-1 $\beta$, IL-6, and IL $13 .^{53}$ Elevation of inflammatory cytokines offers potential therapeutic targets with agents that inhibit cytokine elevations, the use of anti-inflammatory cytokines (such as IL-10), stem cell therapy to re-regulate cytokine dysfunction, and designer drugs of the future that are modeled after these mechanisms. There is still much research to be done to be able to confirm these hopeful clues and observations that play a role in the etiology of autism, especially in patients with immune-associated risk factors or regression-type disease.

Excessive glutaminergic activity has been noted in autism, and may also play a role in inflammatory dysregulation. ${ }^{54,55}$ In addition, alternative practitioners have been discussing the theory that anti-oxidant pathways are insufficient in patients with autism. This relationship has been noted in the past, and evidence of a predisposition of insufficient glutathione or anti-oxidant reserves in autistic patients has been described by James et al. ${ }^{56,57}$ $\mathrm{Chez}^{58}$ previously reported a similar insufficiency in antioxidant protection in patients diagnosed with variants of Landau-Kleffner and concurrent autistic features. Insufficient anti-oxidant protection may not be the initial cause, but rather an endpoint of chronic ongoing inflammation. Supplementation with anti-oxidant promoting compounds, such as $\mathrm{N}$-acetyl cysteine in animal models has helped reverse abnormal cytokine or viral-induced placental inflammation. ${ }^{59}$ Although this may represent reparative or immature responses to neural-glial dysfunction, it may still offer another mechanism that provides hope for therapeutic intervention. Studies of therapies for such neuro-inflammation may have to include techniques for monitoring, including possible use of CSF cytokine measurements. This has yet to be standardized as a commercially available method.

Another exciting category of immune and cytokine research overlapping with autism may involve epileptic EEG patterns seen in young children with autism. As many as $50 \%$ to $60 \%$ of pre-school aged children with autism may develop epileptic spikes on their EEG, es- 
pecially in their sleep. ${ }^{7}$ Researchers in epilepsy, such as Vezzani $^{60}$ and Vezzani and Granata ${ }^{61}$ have shown that cytokine changes may be responsible for development of epileptic activity in animal and human tissue. Certain anti-inflammatory treatments or anti-epileptic drugs may alter some immune mechanisms. There are cases in which the EEG abnormalities and autistic traits have improved in children treated with drugs such as valproic acid. ${ }^{7,62-64}$ Although speculative at this time, further studies are warranted to determine whether ongoing immune dysfunction can be identified by (or correlates with) the eventual onset of epileptic activity on EEG in young children with autism, even in the absence of clinical seizures. The CSF cytokines and neurotransmitters and/or imaging studies would be needed to show correlation to phenotypic features and EEG findings. Once these immune factors are defined and laboratory immune testing for autism may be possible, relationship to epilepsy in autism can be further determined.

\section{PART 2: IMMUNE MODULATION AND INTERVENTIONS}

Immune modulation and therapy, which has been applied to human children or adults with autism, is mainly limited to a number of case reports, unpublished information, and rare case series. Few controlled studies have been done; therefore, defining which therapies offer the best outcomes cannot be interpreted to the highest levels of scientific certainty at this time, and there is a need to consider early pilot study information at best. Without conclusive studies, no consensus treatment guidelines for immune therapies have been developed for physicians to use; there are no agreeable standards for immune-related laboratory investigations of autism. Currently there is no single commercial scientific laboratory that offers immune testing for autism on the basis of any agreed on markers or panels of antibodies or cytokines that could potentially identify a phenotypic subtype of autisticspectrum patients who are good candidates for immunomodulatory-targeted therapy. Measurements of quantitative serum immunoglobulins and IgG subtyping are widely available, including $\mathrm{T}$-cell quantification and type, but these are not specific for autism issues. There are also cytokine panels available, but researchers use a variety of processing and dilution techniques that vary among different research and commercial laboratories; therefore, standardization processes and test procedures have not been clinically established. Study of autistic regression through the use of lumbar puncture and analysis of CSF antibodies and cytokines is not currently a practice widely performed, except in research or on an individual basis by various clinicians around the world. These complicating factors and limited baseline-controlled data in autism research to date have limited the immune research and treatment options described in this section. Therefore, we reviewed limited studies or clinical case reports using peer-reviewed presentations as our best guide to what treatments have been tried and what potential treatments may exist for the future.

\section{Observed improvement in comorbid autoimmune disease and autism}

Case reports of autism have been described with lymphoproliferative disease, nephrotic syndrome, and colitis cases. Treatment with various agents, mainly corticosteroids has been described as helping resolve autism features in cases treated for underlying medical conditions, as previously described. Specifically, one autistic patient with lymphoproliferative disease treated with prednisone showed improvements in speech and social deficits with treatment of the hematologic disease. ${ }^{65}$

Intestinal complaints have been described as a common comorbid condition in autistic patients. Treatment with prednisone and metronidazole has been successful in one case of inflammatory colitis, relieving longstanding social deficits in a patient with high-functioning autism. ${ }^{66}$ Furthermore, in a series of autistic patients with regressive autistic traits and chronic diarrhea, treatment with the antibiotic vancomycin, which may inhibit certain glutamate inflammation effects or anaerobic bacteria, was successful in providing short-term improvement. ${ }^{67}$ These types of cases support the theory that symptoms of autism may be partially reversible. However, long-term outcomes were not reported in these cases.

Pediatric patients with autoimmune conditions, such as Crohn's disease, asthma, nephrotic syndrome, and rheumatoid disease, have already undergone protocols of immune-modulatory therapy. Because there are precedents for pediatric treatment in autoimmune disease and neurological diseases that affect nonautistic children, models of how pediatric patients tolerate different protocols for the use of immunosuppressive agents, such as steroids or other anti-inflammatory medications already exist. These may help in the design of protocols and safety monitoring standards for any future trials of immune-targeted treatments in patients with autism spectrum disorders.

\section{Immunoglobulin therapy}

As mentioned earlier in this article, immunoglobulin deficiencies and increased immune dysfunction exist in autism-spectrum disorders. Trials have been performed in an open label, prospective manner by different researchers. ${ }^{28,29,32,31,68}$ Outcome measurements were mainly limited to subjective observations with some objective parameters. The mechanism of action of intravenous immunoglobulin therapy is believed to work by blocking the production of endogenous auto-antibodies, and downregulating T-cell stimulation of B-cells producing abnormal antibodies. The exact effects in children with autism 
have not been clearly measured, but often those receiving therapy were documented to have lower levels of either total IgG, IgM, or low IgA.

Initially reported by Plioplys ${ }^{32}$ in 1999 , a series of 10 children was observed after receiving $400 \mathrm{mg} / \mathrm{kg}$ of intravenous immunoglobulin (IVIG) and 1 out of 10 children dramatically improved in their autistic findings. Similar results were described in 2000 by $\mathrm{Gupta}^{31}$ and Gupta et $\mathrm{al}^{28}$; in an open-label prospective study, they gave repeated doses of IVIG and improvement occurred in $10 \%$ to $20 \%$ of the patients who were treated. Other authors have also reported a positive IVIG response in autism, but no one has reported curing or totally reversing autism symptoms. ${ }^{68}$ To date, epilepsy studies of IVIG treatment have been confined to use in syndromes of intractable epilepsy. However, in certain conditions like Landau-Kleffner syndrome (LKS) or continuous spike wave in sleep syndrome, IVIG at $400 \mathrm{mg} / \mathrm{kg}$ has been helpful and in rare cases has resulted in resolving the EEG abnormalities and leading to clinical improvement. ${ }^{69,70}$ One must be cautious when comparing continuous spike wave in sleep syndrome or LKS to autism; although language regression may occur in both, most experts recognize that autism-spectrum disorders and these epilepsy conditions are not the same.

\section{Corticosteroids}

Autistic patients have been treated with steroids like adrenocorticotropic hormone (ACTH) or prednisone in the past. The rationale for this was partially based on LKS or continuous spike wave in sleep syndrome and epilepsy, and the observation of EEG improvement response in children with those conditions. Also, because autistic children may have abnormal sleep-activated EEG spikes that resemble the location and other similarities with the more active EEG in LKS, the rationale that similar brain anatomical sites may respond to steroids makes intuitive sense. ${ }^{71}$

Experience with EEG abnormalities and autistic regression cases that respond to steroids have been described in various case reports. ${ }^{62,71-74}$ Treatment was usually prescribed with daily prednisone doses of $2 \mathrm{mg} /$ $\mathrm{kg} /$ day for 3 to 6 months. Limitations to therapy were usually Cushingoid side effects. As in other chronic conditions requiring steroids, pulse dosing was tried with steroids in the form of prednisone or prednisolone at 5 to $10 \mathrm{mg} / \mathrm{kg}$ twice per week. Long-term success with no dependence or minimal Cushingoid effects has been noted in several hundred patients treated in this manner (Chez, unpublished data, personal communication). The majority of studies that assessed the efficacy of steroid treatment included limited objective measurements. However, EEG abnormalities were found to normalize with steroid treatment in $67 \%$ of a large unreported series (360 patients; Chez, unpublished, personal communica- tion). In one series of 36 children studied with magnetoencephalographic evidence, localization of abnormal EEG spikes correlated with a positive response to steroid treatment. If EEG spikes localized to the peri-sylvian region, inferior frontal, or peri-rolandic region, then $85 \%$ of patients responded with predictability, whereas $94 \%$ of nonresponders had more multifocal or widespread spike abnormalities on EEG. ${ }^{75}$ In all, 17 of 32 patients showed response to prednisone after 2 to 4 months of treatment $(53 \%)$. Improvements were seen on EEG and in language skills of the patients. Other steroid treatment series of regressed language in autistic spectrum patients diagnosed with LKS variant showed improved language with pulse-dose steroids. ${ }^{73,76,77}$

A single case report documenting language and social improvement after daily high-dose steroids in a regressive case of autism with normal EEG findings was based on abnormal cortical response to frequency-modulated, auditory-evoked potentials based on work from Stefanatos et al. ${ }^{78}$ This patient had a robust steroid response to prednisone. There has not been any follow-up or similar use of this technique to document steroid response, and although long-term outcome did show improved language and social function, the autism diagnosis remained. In another single case series from the United Kingdom, older teenage autistic patients who were given ACTH injections showed improved social responsiveness. $^{79}$

In summary, among the current studies of immunetargeted therapies, the most collective data on steroid effects on autism is probably the largest. Clear clinical improvements are consistent between different groups that had peer-reviewed assessments. In addition, all reported similar outcomes and side effects were made with the use of steroids. As in IVIG treatment, there has been no report of cure or elimination of all autism features. In the majority of cases, steroid effects did not permanently alter an autism diagnosis in these patients. Clinical concerns about steroid dependency and side effects, such as Cushingoid or long-term, well-known steroid effects have limited more randomized or controlled studies of steroid medications in autism. This is unfortunate, as there may be a potential for significant improvement from steroid treatment on cytokine and chronic immune dysregulation in autism. Future studies may still be warranted.

\section{Treatment of cytokine abnormalities}

Only one study of cytokine levels in CSF and serum in autism has been completed to date. ${ }^{53} \mathrm{~A}$ more recent, open-label trial of six boys with autistic regression and elevated $\mathrm{CSF} /$ serum cytokine profiles looked at treatment using $2.5 \mathrm{mg}$ of lenalidomide per day for 12 weeks. ${ }^{80}$ Lenalidomide (Revlimid; Celgene Corporation, Summit, NJ) is a derivative of thalidomide introduced in 
2004, which was initially intended as a treatment for multiple myeloma. This study measured speech and behavioral outcomes, as well as serum levels and CSF levels, before and after treatment. Elevated baseline levels of TNF- $\alpha$, IL- $1 \beta$, and IL- $1 \alpha$ were observed. All CSF and cytokine levels decreased, with TNF- $\alpha$ and the interleukins showing a statistically significant decrease. After 6 weeks of treatment, improvements in expressive and receptive language, as measured by the Receptive and Expressive One-Word Tests were documented. Reduced symptoms of autism, as measured by the Childhood Autism Rating Scale (CARS) were also noted. Results were not statistically significant, but this was likely due to the small sample size in this phase 2 pilot study. ${ }^{80}$ Regression occurred to some degree in all patients at 2 to 4 weeks after treatment was withdrawn and re-initiation of treatment did seem to reverse this regression (personal communication, Chez). Trials on the use of minocycline to treat patients with cytokine abnormalities in autism are also currently undergoing, but results are not yet available. Treatment with pioglitazone has been described as helping to modulate immune profiles and improve autistic behavior, measured by using the Aberrant Behavioral Checklist. This effect may be due to the immunosuppressive properties of this oral diabetic drug. ${ }^{81}$

Other known cognitive diseases, such as pediatric human immunodeficiency virus dementia and Alzheimer's may involve cytokine dysregulation and elevated TNF- $\alpha$ elevation. ${ }^{82} \mathrm{~A}$ single study of treatment with peri-spinal injection of etanercept, a known TNF- $\alpha$ inhibitor used in rheumatoid arthritis, showed clinical improvements in some daily living skills in Alzheimer's patients. ${ }^{83} \mathrm{~A}$ single case of repetitive regression, with bouts of inflammatory colitis in an 8-year-old with regressive autism after age 3, has shown elevated serum TNF- $\alpha$ levels and rapid colitis, as well as behavioral and language improvements after injections of etanercept (unpublished data, personal communication Y. Davies and M. Chez 2008).

A single, double-blinded, placebo-controlled study of 31 patients (17 placebos, 14 active substance), with Lcarnosine at $800 \mathrm{mg}$ per day for 12 weeks, showed a clinically significant improvement in receptive language of autistic patients with regression. This was represented by a mean change on the Receptive One-Word Picture Vocabulary test (age adjusted) from baseline to 8 weeks of $40.57 \pm 24.55$ to $47.86 \pm 28.37(p<0.02)$ in the treated group. The placebo group showed no statistically or clinically significant changes. ${ }^{47}$ This supplement has known neuroprotective properties, including blocking zinc and copper influx with cell death in ischemia, and inhibiting TNF- $\alpha$. Unfortunately, no CSF or serum cytokine profiles were available from patients in this study.

Abnormal glial-modulated inflammatory responses can be found with dysregulation of expected cytokine levels of TNF- $\alpha$ and IL-1 $\beta$. The anti-convulsant, valproic acid, which may modulate interleukin-1 levels, is used as a mood stabilizer and anti-epileptic drug in studies of autism. Its success in treating these symptoms in autistic patients may reveal another mechanism by which valproic acid works. Cases of improvement in autistic regression or significant EEG and clinical improvement have been reported by at least three authors using valproic acid. ${ }^{62,64,79,84}$ This drug also has extensive placebocontrolled studies in autism showing improved behavior in autistic patients' moods and behaviors, especially when abnormal EEG is present in the patient. ${ }^{85}$ Further examination of the action of drugs, such as valproic acid on cytokine profiles, may be useful. It is also known that fetal exposure to valproic acid can cause autism features in animal models, and can also affect mental development in offspring of pregnant epileptic women on this medication. ${ }^{86}$ This may be related to the timing of cytokine effects in utero because the postnatal effects that are known to be different in the human brain show this medication to be beneficial in autism after the perinatal period of development is complete.

\section{Glutamate and NMDA receptors}

A common endpoint for glial and CNS immune response would be elevated glutamate levels and cell death. NMDA receptors may play a significant role in a disrupted, chronically aggravated innate immune dysregulation. Prior reports of elevated glutamate levels and receptors in the autistic brain have been reported. ${ }^{55}$ Modulation of NMDA receptors by NMDA antagonists in autism has been reported with noted clinical improvements. D-cycloserine, an antibiotic that also binds NMDA receptors has been shown to improve behavioral functions in autism. ${ }^{87}$ Studies of memantine, an NMDA antagonist, have shown significant improvements in autism in at least two reported studies, with one study that had almost 200 patients. ${ }^{88,89}$ These observations have led to memantine studies that are currently in progress with the potential hope of future clinical application in autism. Amantidine has also been studied as a potential treatment in autism, as it also acts on the NMDA receptor. ${ }^{88,90}$

\section{Anti-oxidant protection}

Evidence of poor glutathione levels and deficient superoxide dismutase levels have been described in various chronic neurological conditions including autism. ${ }^{56-58}$ Evidence that supplements like $\mathrm{N}$-acetyl cysteine may alter placental inflammation have been reported. ${ }^{91}$ This compound also offers anti-oxidant protection. Studies of $\mathrm{N}$-acetyl cysteine in obsessive compulsive disorders have also been reported, which are believed to act on glutamate receptors. ${ }^{91}$ No publications of glutathione injections for autism have been reported in a published case series, and only anecdotal evidence is available. 
Anti-oxidant protection can occur with L-carnosine, and this compound has already been previously discussed in the section on possible cytokine-modulation effects.

\section{Stem cells}

The potential of stem cell therapy may be most interesting in the modulating capacity as discussed in previous sections. This type of treatment may allow specific immune programming of stem cells, which may lead to correction of disrupted innate CNS or systemic immunity. At this time, no studies in autism have been done. Stem cells are expected to be researched in the future as potential autism therapy by hypothetically targeting the immune system.

\section{CONCLUSIONS}

Immune dysregulation in autism-spectrum disorders may affect a large proportion of those with this diagnosis, especially those who regress. This may influence other factors, such as the risk of an abnormal EEG. Possible genetic or environmental factors may influence innate CNS immunity. The latest hypotheses seem to suggest possible in utero maternal factors that act transplacentally with auto-antibodies or cytokine dysregulation leading to neurodevelopmental changes that may not become evident until well past the first 9 to 12 months of life. This may be partially or completely modulated by possible immunomodulating therapies. Targeting such designer drugs may offer future hope for modifying the course of autism due to theoretical immune dysregulation. This assumes standardized testing for markers of maternal or infant risk factors may be available in the future.

Current immune therapies and drugs that may work have been discussed. Some drugs, such as valproic acid and memantine, have already been shown to improve some aspects of autism. Corticosteroid experience has shown promising transient and sometimes stable improvement, but clearly more specific drugs with less broad side-effect risks are needed. Less impressive results with immunoglobulins have also shown that this type of immunomodulatory therapy can help some autistic patients gain some language or behavioral improvement; however, this is much more costly than steroid treatment for a less robust effect. Cytokine levels may be critically important in the developing brain, as well as in reversing some or all of the symptoms of autistic regression. At this time, more standardized study and knowledge is needed to determine whether cytokine dysregulation is the cause, or just another system that is dysfunctional in some forms of autism.

Future therapies will potentially include some type of cytokine modulator, and perhaps prenatal interventions that will correct the in utero immune experience for the potentially harmed fetus. More research in these mechanisms will be needed before such therapies can be fully developed. Currently, there are no standardized recommendations as to which therapies that help the most can be made. Limitations to date include the lack of consensus on mechanism, limited prior placebo controlled trials, and mainly the observational nature of evidence in studies of autistic patients. Further studies in both animal models and human patients are desperately needed. For now, the benefit of individualized treatment of suspected immune problems needs to be determined on a case by case basis, and must be used to intervene when believed to be medically appropriate by the treating physician.

\section{REFERENCES}

1. Rice C. Prevalence of autism spectrum disorders. Autism and Developmental Disabilities Monitoring Network, United States, 2006. MMWR 2009;58;1.

2. American Psychiatric Association: Diagnostic and Statistical Manual of Mental Disorders, 4th edition, text revision. Washington, DC: Skyscape, 1994

3. Shattuck PT. The contribution of diagnostic substitution to the growing administrative prevalence of autism in US special education. Pediatrics 2006;117:1028-1037.

4. Department of Developmental Services. Autistic spectrum disorders: changes in the california caseload: an update 1999-2002. Sacramento, CA: State of California; 2003.

5. Croen LA, Grether JK, Hoogstrate J, et al. The changing prevalence of autism in California. J Autism Dev Disord 2002;32:207215

6. Institute MIND. Report to the legislature on the principle findings from the epidemiology of autism in California. Sacramento, CA: University of California, Davis, 2002

7. Chez MG, Chang M, Krasne V, et al. Frequency of epileptiform EEG abnormalities in a sequential screening of autistic patients with no known clinical epilepsy from 1996 to 2005. Epilepsy Behav 2006;8:267-271.

8. Olsson I, Steffenburg S, Gillberg C. Epilepsy in autism and autisticlike conditions. A population-based study. Arch Neurol 1988; 45:666-668

9. Spence SJ. The genetics of autism. Semin Pediatr Neurol 2004;11: 196-204.

10. Wassink TH, Piven J, Patil SR. Chromosomal abnormalities in a clinic sample of individuals with autistic disorder. Psychiatr Genet 2001;11:57-63.

11. Campbell DB, Sutcliffe JS, Ebert PJ, et al. A genetic variant that disrupts MET transcription is associated with autism. Proc Natl Acad Sci U S A 2006;103:16834-16839.

12. Patterson P. Pregnancy, immunity, schizophrenia, and autism. Eng Sci 2006;69:10-21.

13. Connolly AM, Chez MG, Pestronk A, et al. Serum auto-antibodies to brain in Landau-Kleffner variant, autism, and other neurologic disorders. J Pediatr 1999;134:607-613.

14. Nelson KB, Grether JK, Croen LA, et al. Neuropeptides and neurotrophins in neonatal blood of children with autism or mental retardation. Ann Neurol 2001;49:597-606.

15. Pardo CA, Vargas DL, Zimmerman AW. Immunity, neuroglia and neuroinflammation in autism. Int Rev Psychiatry 2005;17:485495.

16. Todd RD, Hickok JM, Anderson GM, et al. Antibrain antibodies in infantile autism. Biol Psychiatry 1988;23:644-647.

17. Vargas DL, Nascimbene C, Krishnan C, et al. Neuroglial activation and neuroinflammation in the brain of patients with autism. Ann Neurol 2005;57:67-81.

18. Atladottir HO, Pedersen MG, Thorsen P, et al. Association of family history of autoimmune diseases and autism spectrum disorders. Pediatrics 2009;124:687-694. 
19. Comi AM, Zimmerman AW, Frye VH, et al. Familial clustering of autoimmune disorders and evaluation of medical risk factors in autism. J Child Neurol 1999;14:388-394.

20. Daniels WW, Warren RP, Odell JD, et al. Increased frequency of the extended or ancestral haplotype B44-SC30-DR4 in autism. Neuropsychobiology 1995;32:120-123.

21. Warren RP, Margaretten NC, Pace NC, et al. Immune abnormalities in patients with autism. J Autism Dev Disord 1986;16:189197.

22. Connolly AM. Brain-derived neurotrophic factor and auto-antibodies to neural antigens in sera of children with autistic spectrum disorders, Landau-Kleffner syndrome, and epilepsy. Biol Psychiat 2006;59:354-363.

23. Singh VK, Lin SX, Yang VC. Serological association of measles virus and human herpesvirus- 6 with brain auto-antibodies in autism. Clin Immunol Immunopathol 1998;89:105-108.

24. Singh VK, Warren RP, Odell JD, et al. Antibodies to myelin basic protein in children with autistic behavior. Brain Behav Immun 1993; 7:97-103.

25. Warren RP, Yonk LJ, Burger RA, et al. Deficiency of suppressorinducer $(\mathrm{CD} 4+\mathrm{CD} 45 \mathrm{RA}+) \mathrm{T}$ cells in autism. Immunol Invest 1990;19:245-251.

26. Stubbs EG, Crawford ML. Depressed lymphocyte responsiveness in autistic children. J Autism Child Schizophr 1977;7:49-55.

27. Margaretten NC, Warren RP. Reduced natural killer cell activity and OKT4/OKT8 ratio in epileptic patients. Immunol Invest 1986; 15:159-167.

28. Gupta S, Aggarwal S, Heads C. Dysregulated immune system in children with autism: beneficial effects of intravenous immune globulin on autistic characteristics. J Autism Dev Disord 1996;26: 439-452.

29. Plioplys A. Immunoglobulin reactivity in autism and Rett's syndrome. Dev Brain Dysfunct 1994;7:12-16.

30. Zimmerman AW. Serum immunoglobulins and autoimmune profiles in children with autism. Ann Neurol 1995;38:528.

31. Gupta S. Treatment of children with autism with intravenous immunoglobulin. J Child Neurol 1999;14:203-205.

32. Plioplys A. Intravenous immunoglobulin treatment of children with autism. J Child Neurol 1999;13:79-82.

33. Risch N, Spiker D, Lotspeich L, et al. A genomic screen of autism: evidence for a multilocus etiology. Am J Hum Genet 1999;65:493507.

34. Bailey A, Le Couteur A, Gottesman I, et al. Autism as a strongly genetic disorder: evidence from a British twin study. Psychol Med 1995;25:63-77.

35. Hallmayer J, Glasson EJ, Bower C, et al. On the twin risk in autism. Am J Hum Genet 2002;71:941-946.

36. Steffenburg S, Gillberg C, Hellgren L, et al. A twin study of autism in Denmark, Finland, Iceland, Norway and Sweden. J Child Psychol Psych 1989;30:405-416.

37. Boccone L, Dessi V, Zappu A, et al. Bannayan-Riley-Ruvalcaba syndrome with reactive nodular lymphoid hyperplasia and autism and a PTEN mutation. Am J Med Genet A 2006;140:1965-1969.

38. Burger RA. Possible immunogenetic basis for autism. Ment Retard Dev D R 1998;4:137-141.

39. Ashwood P, Wills S, Van de Water J. The immune response in autism: a new frontier for autism research. J Leukoc Biol 2006; 80:1-15.

40. Shi L, Fatemi SH, Sidwell RW, et al. Maternal influenza infection causes marked behavioral and pharmacological changes in the offspring. J Neurosci 2003;23:297-302.

41. Nawa H, Takei N. Recent progress in animal modeling of immune inflammatory processes in schizophrenia: implication of specific cytokines. Neurosci Res 2006;56:2-13.

42. Palmen SJ, van Engeland H, Hof PR, et al. Neuropathological findings in autism. Brain 2004;127(Pt 12):2572-2583.

43. Patterson PH. Modeling features of autism in animals. Boca Raton, FL: Taylor \& Francis, 2005

44. Smith SE, Li J, Garbett K, et al. Maternal immune activation alters fetal brain development through interleukin-6. J Neurosci 2007; 27:10695-10702.

45. Urakubo A, Jarskog LF, Lieberman JA, et al. Prenatal exposure to maternal infection alters cytokine expression in the placenta, amniotic fluid, and fetal brain. Schizophr Res 2001;47:27-36.

46. Meyer U, Murray PJ, Urwyler A, et al. Adult behavioral and pharmacological dysfunctions following disruption of the fetal brain balance between pro-inflammatory and IL-10-mediated antiinflammatory signaling. Mol Psychiatry 2008;13:208-221.

47. Chez MG, Buchanan CP, Aimonovitch MC, et al. Double-blind, placebo-controlled study of L-carnosine supplementation in children with autistic spectrum disorders. J Child Neurol 2002;17:833837.

48. Morris C. Fetal anti-brain anti-bodies in serum of mothers of children with autistic disorder: correlation with developmental regression. Ann Neurol 2007;62:S116-S117.

49. Morris C. Maternal antibodies and placental-fetal IgG transfer theory. Totowa, NJ: Humana Press, 2008.

50. Singer HS. Serum anti-brain antibody differences in mothers of children with autism disorder: a study with fetal human and rodent tissue. International Meeting for Autism Research (IMFAR), 2007.

51. Zimmerman AW, Connors SL, Matteson KJ, et al. Maternal antibrain antibodies in autism. Brain Behav Immun 2007;21:351-357.

52. Croen LA, Grether JK, Yoshida CK, et al. Maternal autoimmune diseases, asthma and allergies, and childhood autism spectrum disorders: a case-control study. Arch Pediatr Adolesc Med 2005; 159:151-157.

53. Chez MG, Dowling T, Patel PB, et al. Elevation of tumor necrosis factor-alpha in cerebrospinal fluid of autistic children. Pediatr Neurol 2007;36:361-365.

54. Jander S, Schroeter M, Stoll G. Role of NMDA receptor signaling in the regulation of inflammatory gene expression after focal brain ischemia. J Neuroimmunol 2000;109:181-187.

55. Purcell AE, Jeon OH, Zimmerman AW, et al. Postmortem brain abnormalities of the glutamate neurotransmitter system in autism. Neurology 2001;57:1618-1628.

56. James SJ, Cutler P, Melnyk S, et al. Metabolic biomarkers of increased oxidative stress and impaired methylation capacity in children with autism. Am J Clin Nutr 2004;80:1611-1617.

57. James SJ, Slikker W, 3rd, Melnyk S, et al. Thimerosal neurotoxicity is associated with glutathione depletion: protection with glutathione precursors. Neurotoxicology 2005;26:1-8.

58. Chez MG. Superoxide dismutase overactivity, excessive selenium, and low copper in acquired epileptic aphasia (the Landau-Kleffner syndrome). Ann Neurol 1995;39:544.

59. Beloosesky R, Gayle DA, Amidi F, et al. N-acetyl-cysteine suppresses amniotic fluid and placenta inflammatory cytokine responses to lipopolysaccharide in rats. Am J Obstet Gynecol 2006; 194:268-273.

60. Vezzani A. Inflammation and epilepsy. Epilepsy Curr 2005;5:1-6.

61. Vezzani A, Granata T. Brain inflammation in epilepsy: experimental and clinical evidence. Epilepsia 2005;46:1724-1743.

62. Chez MG. Treatment of electroencephalographic epileptiform activity on overnight EEG studies in children with pervasive developmental disorder or autism: defining similarities to the LandauKleffner syndrome. J Dev Learning Disord 1998;2:217-229.

63. Hollander E, Dolgoff-Kaspar R, Cartwright C, et al. An open trial of divalproex sodium in autism spectrum disorders. J Clin Psychiatry 2001;62:530-534.

64. Plioplys A. Autism: electroencephalogram abnormalities and clinical improvement with valproic acid. Arch Pediat Adol Med 1994; 148:220-222.

65. Shenoy S, Arnold S, Chatila T. Response to steroid therapy in autism secondary to autoimmune lymphoproliferative syndrome. J Pediatr 2000;136:682-687.

66. Sandler RH, Bolte ER, Chez MG, et al. Relief of psychiatric symptoms in a patient with Crohn's disease after metronidazole therapy. Clin Infect Dis 2000;30:213-214.

67. Sandler RH, Finegold SM, Bolte ER, et al. Short-term benefit from oral vancomycin treatment of regressive-onset autism. J Child Neurol 2000;15:429-435.

68. Boris M. Improvement in children with autism treated with intravenous gamma globulin. J Nutr Environ Med 2005;15:169-176.

69. Arts WF, Aarsen FK, Scheltens-de Boer M, et al. Landau-Kleffner syndrome and CSWS syndrome: treatment with intravenous immunoglobulins. Epilepsia 2009;50(Suppl 7):55-58. 
70. Mikati MA, Saab R. Successful use of intravenous immunoglobulin as initial monotherapy in Landau-Kleffner syndrome. Epilepsia 2000;41:880-886.

71. Lewine JD, Andrews R, Chez M, et al. Magnetoencephalographic patterns of epileptiform activity in children with regressive autism spectrum disorders. Pediatrics 1999;104(3 Pt 1):405-418.

72. Chez M. Practical treatment with pulse-dose corticosteroids in pervasive developmental disorder of autistic patients with abnormal sleep EEG and language delay. In: Perat M, ed. New developments in child neurology. Bologna, Italy: Monduzzi Editore, 1998.

73. Chez M. Pulse high-dose steroids as combination therapy with valproic acid in epileptic aphasia patients with pervasive developmental delay or autism. Ann Neurol 1998;44:539.

74. Trauner D. Developmental aphasia with epileptiform abnormalities on EEG: clinical features and response to Prednisone. Ann Neurol 2002;52:66-67.

75. Lewine JD. Patterns of epileptiform activity and clinical response to steroid therapy in autism. International Meeting for Autism Research (IMFAR). Chicago, IL, 2009.

76. Aykut-Bingol C. Pulse methylprednisolone therapy in LandauKleffner syndrome. J Epilepsy 1998;9:189-191.

77. Hempel A. Treatment of Landau-Kleffner syndrome with pulse dose prednisone: behavioral and language outcomes. Boston, MA: American Epilepsy Society Meeting, 2009.

78. Stefanatos GA, Grover W, Geller E. Case study: corticosteroid treatment of language regression in pervasive developmental disorder. J Am Acad Child Adolesc Psychiatry 1995;34:1107-1111.

79. Buitelaar JK, van Engeland H, de Kogel KH, et al. The use of adrenocorticotrophic hormone (4-9) analog ORG 2766 in autistic children: effects on the organization of behavior. Biol Psychiat 1992;31:1119-1129.

80. Chez M. Effect of Lenalidomide on TNF-alpha elevation and behavior in autism. Presented at: International Meeting for Autism Research (IMFAR); May 20-22, 2010; Philadelphia, PA.

81. Boris M, Kaiser CC, Goldblatt A, et al. Effect of pioglitazone treatment on behavioral symptoms in autistic children. J Neuroinflammation 2007;4:3.
82. Brabers NA, Nottet HS. Role of the pro-inflammatory cytokines TNF-alpha and IL-1beta in HIV-associated dementia. Eur J Clin Invest 2006;36:447-458.

83. Tobinick EL, Gross H. Rapid cognitive improvement in Alzheimer's disease following perispinal etanercept administration. J Neuroinflammation 2008;5:2.

84. Deonna T. Reversible Behavioral autistic-like regression: a manifestation of a special (new?) Epileptic syndrome in a 28 month old child. A 2 year longitudinal study. Neurocase 1995; 1:91-95.

85. Hollander E, Chaplin W, Soorya L, et al. Divalproex sodium vs placebo for the treatment of irritability in children and adolescents with autism spectrum disorders. Neuropsychopharmacol 2010;35: 990-998.

86. Meador KJ, Baker GA, Browning N, et al. Cognitive function at 3 years of age after fetal exposure to antiepileptic drugs. N Engl J Med 2009;360:1597-1605.

87. Posey DJ, Kem DL, Swiezy NB, et al. A pilot study of Dcycloserine in subjects with autistic disorder. Am J Psychiatry 2004;161:2115-2117.

88. King B. Double-blind, placebo-controlled study of amantidine hydrochloride in the treatment of children with autistic disorder. J Am Acad Child Psy 2001;184:254-256.

89. Owley T, Salt J, Guter S, et al. A prospective, open-label trial of memantine in the treatment of cognitive, behavioral, and memory dysfunction in pervasive developmental disorders. J Child Adolesc Psychopharmacol 2006;16:517-524.

90. Chez MG, Burton Q, Dowling T, et al. Memantine as adjunctive therapy in children diagnosed with autistic spectrum disorders: an observation of initial clinical response and maintenance tolerability. J Child Neurol 2007;22:574-579.

91. Lafleur DL, Pittenger C, Kelmendi B, et al. N-acetylcysteine augmentation in serotonin reuptake inhibitor refractory obsessivecompulsive disorder. Psychopharmacology (Berl) 2006;184:254256. 